OPEN ACCESS

Edited by:

Cyrus Khandanpour,

University Hospital Münster, Germany

Reviewed by:

Angela Fleischman,

University of California, Irvine,

United States

Nicolas Chatain

University Hospital Aachen, Germany

Jonathan Tan,

Bond University, Australia

*Correspondence:

Huichun Zhan

Huichun.Zhan@

stonybrookmedicine.edu

Specialty section: This article was submitted to Hematologic Malignancies,

a section of the journal

Frontiers in Oncology

Received: 04 August 2021 Accepted: 07 October 2021 Published: 26 October 2021

Citation:

Zhang $H$, Yeware $A$, Lee $S$ and Zhan H (2021) A Murine Model With JAK2V617F Expression in Both Hematopoietic Cells and Vascular Endothelial

Cells Recapitulates the Key

Features of Human Myeloproliferative Neoplasm.

Front. Oncol. 11:753465. doi: 10.3389/fonc.2021.753465

\section{A Murine Model With JAK2V617F Expression in Both Hematopoietic Cells and Vascular Endothelial Cells Recapitulates the Key Features of Human Myeloproliferative Neoplasm}

\author{
Haotian Zhang ${ }^{1}$, Amar Yeware ${ }^{2}$, Sandy Lee ${ }^{3}$ and Huichun Zhan ${ }^{2,4 *}$ \\ ${ }^{1}$ Graduate Program in Molecular \& Cellular Biology, Stony Brook University, Stony Brook, NY, United States, ${ }^{2}$ Department of \\ Medicine, Stony Brook School of Medicine, Stony Brook, NY, United States, ${ }^{3}$ Graduate Program in Molecular \& Cellular \\ Pharmacology, Stony Brook University, Stony Brook, NY, United States, ${ }^{4}$ Medical Service, Northport VA Medical Center, \\ Northport, NY, United States
}

The myeloproliferative neoplasms (MPNs) are characterized by an expansion of the neoplastic hematopoietic stem/progenitor cells (HSPC) and an increased risk of cardiovascular complications. The acquired kinase mutation JAK2V617F is present in hematopoietic cells in a majority of patients with MPNs. Vascular endothelial cells (ECs) carrying the JAK2V617F mutation can also be detected in patients with MPNs. In this study, we show that a murine model with both JAK2V617F-bearing hematopoietic cells and JAK2V617F-bearing vascular ECs recapitulated all the key features of the human MPN disease, which include disease transformation from essential thrombocythemia to myelofibrosis, extramedullary splenic hematopoiesis, and spontaneous cardiovascular complications. We also found that, during aging and MPN disease progression, there was a loss of both HSPC number and HSPC function in the marrow while the neoplastic hematopoiesis was relatively maintained in the spleen, mimicking the advanced phases of human MPN disease. Different vascular niche of the marrow and spleen could contribute to the different JAK2V617F mutant stem cell functions we have observed in this JAK2V617F-positive murine model. These results indicate that the spleen is functionally important for the JAK2V617F mutant neoplastic hematopoiesis during aging and MPN disease progression. Compared to other MPN murine models reported so far, our studies demonstrate that JAK2V617F-bearing vascular ECs play an important role in both the hematologic and cardiovascular abnormalities of MPN.

Keywords: myeloproliferative neoplasm, cardiovascular diseases, endothelial cells, JAK2V617F, murine model 


\section{HIGHLIGHTS}

- A murine model in which JAK2V617F is expressed in both hematopoietic cells and ECs recapitulated the key features of the human MPN disease

- Different vascular niche of the marrow and spleen could contribute to different JAK2V617F mutant HSC functions during MPN disease progression

\section{INTRODUCTION}

The Philadelphia chromosome-negative myeloproliferative neoplasms (MPNs), which include polycythemia vera (PV), essential thrombocythemia (ET), and primary myelofibrosis (PMF), are clonal stem cell disorders characterized by hematopoietic stem/progenitor cell (HSPC) expansion, overproduction of mature blood cells, a tendency to extramedullary hematopoiesis, an increased risk of transformation to acute leukemia or myelofibrosis, and an increased risk of vascular thrombosis $(1,2)$. The incidence of MPNs increases significantly with aging and MPN is uncommon before the age of 50 years (3). Older age and longer disease duration are also associated with higher risk of disease transformation to myelofibrosis or secondary acute myeloid leukemia, as well as increased morbidity and mortality in these patients (4). These observations suggest that aging plays an important role in MPN development.

The acquired signaling kinase mutation $J A K 2 V 617 F$ is present in most patients with MPNs and aberrant JAK-STAT signaling plays a central role in these disorders (5). Although JAK2V617F-positive murine models have provided unequivocal evidence that JAK2V617F is able to cause MPNs, there is significant heterogeneity in disease phenotypes between different murine models, and none has been able to recapitulate both the myeloproliferative phenotype and the cardiovascular pathology in patients with MPNs (6). In addition, these murine models were mostly followed for less than 3-9 months (7-18) and how aging affects MPN disease progression has not been studied.

Endothelial cells (ECs) are an essential component of the hematopoietic niche and most HSPCs reside close to a marrow sinusoid (the "perivascular niche") (19). Vascular ECs also play critical roles in the regulation of hemostasis and thrombosis (20). The JAK2V617F mutation can be detected in microvascular ECs isolated from liver and spleen (by laser microdissection), and marrow (by flow cytometry sorting) in $60-70 \%$ of patients with MPNs $(21,22)$. The mutation can also be detected in $60-80 \%$ of EC progenitors derived from the hematopoietic lineage and, in some reports based on in vitro culture assays, in endothelial colony-forming cells from patients with MPNs (22-26). Previously, we reported that the JAK2V617F-bearing vascular endothelium promotes the expansion of the JAK2V617F mutant HSPCs in preference to wild-type HSPCs (27-31) and contributes to the development of cardiovascular complications (32) in a murine model of MPN. In the present study, we investigated how MPN progresses in the JAK2V617F-bearing vascular niche during aging.

\section{MATERIALS AND METHODS}

\section{Experimental Mice}

JAK2V617F Flip-Flop (FF1) mice (12) was provided by Radek Skoda (University Hospital, Basal, Switzerland) and Tie2-Cre mice (33) by Mark Ginsberg (University of California, San Diego). FF1 mice were crossed with Tie2-Cre mice to express $J A K 2 V 617 F$ specifically in all hematopoietic cells (including HSPCs) and vascular ECs (Tie ${ }^{+/-} \mathrm{FF}^{+/-}$, or Tie2FF1), so as to model the human diseases in which both the hematopoietic stem cells and ECs harbor the mutation. All mice used were crossed onto a C57BL/6 background and bred in a pathogen-free mouse facility at Stony Brook University. Animal experiments were performed in accordance with the guidelines provided by the Institutional Animal Care and Use Committee.

\section{Marrow and Spleen Cell Isolation}

Murine femurs and tibias were first harvested and cleaned thoroughly. Marrow cells were flushed into PBS with 2\% fetal bovine serum using a $25 \mathrm{G}$ needle and syringe. Remaining bones were crushed with a mortar and pestle followed by enzymatic digestion with DNase I $(25 \mathrm{U} / \mathrm{ml})$ and Collagenase D $(1 \mathrm{mg} / \mathrm{ml})$ at $37^{\circ} \mathrm{C}$ for $20 \mathrm{~min}$ under gentle rocking. Tissue suspensions were thoroughly homogenized by gentle and repeated mixing using $10 \mathrm{ml}$ pipette to facilitate dissociation of cellular aggregates. Resulting cell suspensions were then filtered through a $40 \mathrm{uM}$ cell strainer.

Murine spleens were collected and placed into a $40 \mathrm{uM}$ cell strainer. The plunger end of a $1 \mathrm{ml}$ syringe was used to mash the spleen through the cell strainer into a collecting dish. $5 \mathrm{ml}$ PBS with $2 \%$ FBS was used to rinse the cell strainer and the resulting spleen cell suspension was passed through a $5 \mathrm{ml}$ syringe with a $23 \mathrm{G}$ needle several times to further eliminate small cell clumps.

\section{Complete Blood Counts and In Vitro Assays}

Complete blood counts and hematopoietic colony formation assays were performed as we previously described (34). Mouse methylcellulose complete media (Stem Cell Technologies, Vancouver, BC) was used to assay hematopoietic colony formation, which was enumerated according to the manufacturer's protocol.

For Lineage negative ( $\left.\mathrm{Lin}^{-}\right)$cell culture, marrow or splenic Lin $^{-}$cells were first enriched using the Lineage Cell Depletion Kit (Miltenyi Biotec). On Day 0, 1,000 Lin $^{-}$cells were seeded in a 48well plate and cultured in 150ul StemSpan serum-free expansion medium (SFEM) containing recombinant mouse Stem cell factor $(100 \mathrm{ng} / \mathrm{ml})$, recombinant mouse Interleukin-3 $(6 \mathrm{ng} / \mathrm{ml})$ and recombinant human Interleukin-6 $(10 \mathrm{ng} / \mathrm{ml})$ (all from Stem Cell Technologies). 200ul fresh SFEM medium with cytokines was added on Day 5 and 8 and cells were counted on Day 5 and 10 . 


\section{Histology}

Femur and spleen tissues were fixed in cold $4 \%$ paraformaldehyde for $6 \mathrm{hr}$ at $4^{\circ} \mathrm{C}$ while shaking. The tissues were washed with PBS for 8 -16hrs at room temperature to remove paraformaldehyde. Femurs were then decalcified and paraffin sections $(5-\mu \mathrm{m}$ thickness) were stained with hematoxylin and eosin or reticulin (Reticulum II Staining Kit, Roche, Tucson, AZ) to assess fibrosis. Images were taken using a Nikon Eclipse TS2R inverted microscope (Nikon, Melville, NY).

\section{Flow Cytometry}

All samples were analyzed by flow cytometry using a FACSAriaTM III or a LSR II (BD biosciences, San Jose, CA, USA). Lineage cocktail (include CD3, B220, Gr1, CD11b, Ter119; Biolegend), cKit (Clone 2B8, Biolegend), Sca1 (Clone D7, Biolegend), CD150 (Clone mShad150, eBioscience), CD48 (Clone HM48-1, Biolegend), CD45 (Clone 104) (Biolegend, San Diego, CA, USA), and CD31 (Clone 390, BD biosciences) antibodies were used.

\section{BrdU Incorporation Analysis}

Mice were injected intraperitoneally with a single dose of 5bromo-2'-deoxyuridine (BrdU; $100 \mathrm{mg} / \mathrm{kg}$ body weight) and maintained on $1 \mathrm{mg} \mathrm{BrdU/ml} \mathrm{drinking} \mathrm{water} \mathrm{for} \mathrm{two} \mathrm{days.} \mathrm{Mice}$ were then euthanized and marrow cells isolated as described above. For analysis of HSC ( $\mathrm{Lin}^{-} \mathrm{CKit}^{+} \mathrm{Sca} 1^{+} \mathrm{CD} 150^{+} \mathrm{CD} 48^{-}$) proliferation, $\mathrm{Lin}^{-}$cells were first enriched using the Lineage Cell Depletion Kit (Miltenyi Biotec) before staining with fluorescent antibodies specific for cell surface HSC markers, followed by fixation and permeabilization using the Cytofix/Cytoperm kit (BD Biosciences, San Jose, CA), DNase digestion (Sigma, St. Louis, MO), and anti-BrdU antibody (Biolegend, San Diego, CA) staining to analyze BrdU incorporation (31).

\section{Analysis of Apoptosis by Active Caspase-3 Staining}

Marrow cells were stained with fluorescent antibodies specific for cell surface HSC markers, followed by fixation and permeabilization using the Cytofix/Cytoperm kit (BD Biosciences). Cells were then stained using a rabbit antiactivated caspase-3 antibody (31). Data were acquired using a LSR II flow cytometer.

\section{Analysis of Senescence by Senescence Associated $\beta$-Galactosidase Activity}

Marrow cells were stained with fluorescent antibodies specific for cell surface HSC markers. Cells were then washed and fixed using $2 \%$ paraformaldehyde and incubated with CellEvent ${ }^{\text {TM }}$ Senescence Green Probe (ThermoFisher Scientific, Waltham, MA) according to the manufacturer's instruction. Data were acquired using a LSR II flow cytometer.

\section{VE-Cadherin In Vivo Staining and Immunofluorescence Imaging}

25ug Alexa Fluor 647-conjugated monoclonal antibodies that target mouse VE-cadherin (clone BV13, Biolegend) were injected retro-orbitally into $2 \mathrm{yr}$ old Tie2FF1 or control mice under anesthesia (35). Ten minutes after antibody injection, the mice were euthanized. Mouse femurs and spleens were dissected out and washed in PBS. After fixation in $4 \%$ paraformaldehyde (PFA) (Affymetrix) for $6 \mathrm{hr}$ at $4^{\circ} \mathrm{C}$ while rotating, the samples were washed in PBS overnight to remove PFA, cryoprotected in $20 \%$ sucrose, embedded in OCT compound (Tissue-Tek), and flash frozen at $-80^{\circ} \mathrm{C}$. Frozen samples were cryosectioned $(\sim 10 \mathrm{uM})$ using a Leica CM1510S Cryostat. Images were acquired using a Nikon Eclipse Ts2R inverted fluorescence microscope.

\section{Transthoracic Echocardiography}

Transthoracic echocardiography was performed on mildly anesthetized spontaneously breathing mice (sedated by inhalation of $1 \%$ isoflurane, $1 \mathrm{~L} / \mathrm{min}$ oxygen), using a Vevo 3100 high-resolution imaging system (VisualSonics Inc., Toronto, Canada). Both parasternal long-axis and sequential parasternal short-axis views were obtained to assess global and regional wall motion. Left ventricular (LV) dimensions at endsystole and end-diastole and fractional shortening (percent change in LV diameter normalized to end-diastole) were measured from the parasternal long-axis view using linear measurements of the LV at the level of the mitral leaflet tips during diastole. LV ejection fraction (EF), volume, and mass are measured and calculated using standard formulas for the evaluation of LV systolic function $(32,36)$.

\section{Histology}

Hearts and lungs were fixed in $4 \%$ PFA overnight at $4^{\circ} \mathrm{C}$ while rotating. The tissues were then washed multiple times with PBS at room temperature to remove PFA. Paraffin sections $(5-\mu \mathrm{m}$ thickness) were stained with Hematoxylin/Eosin (H\&E) following standard protocols. Images were taken using a Nikon Eclipse Ts2R inverted microscope.

\section{Statistical Analysis}

Statistical analyses were performed using Student's unpaired, 2tailed $t$ tests using Excel software (Microsoft). A $p$ value of less than 0.05 was considered significant. For all bar graphs, data are presented as mean \pm standard error of the mean (SEM).

\section{RESULTS}

\section{The Tie2FF1 Mice Develop ET to PMF Transformation During Aging}

To study the effects of the JAK2V617F-bearing vascular niche on MPN disease development in vivo, we crossed mice that bear a Cre-inducible human JAK2V617F gene (FF1) (12) with Tie2-Cre mice (33) to express $J A K 2 \mathrm{~V} 617 \mathrm{~F}$ specifically in all hematopoietic cells and ECs (Tie2FF1). The Tie2FF1 mice developed an ET-like phenotype with neutrophilia (3.8 vs $\left.\left.1.8 \times 10^{3}\right) / \mathrm{uL}, P=0.014\right)$, thrombocytosis (1068 vs $558 \times 10^{3} / \mathrm{uL}, P=0.036$ ), and normal hemoglobin at $2 \mathrm{mo}$ of age, results consistent with previous reports $(28,37)$. We followed these mice up to $18 \mathrm{mo}$ of age to evaluate how the $J A K 2 V 617 F$ mutant vascular niche regulate 
MPN neoplastic hematopoiesis and disease transformation during aging. The Tie2FF1 mice continued to develop increasing neutrophilia and thrombocytosis, both of which plateaued at $\sim 1$ yr of age. In addition, the mice developed significant lymphocytosis and anemia after $6 \mathrm{mo}$ of age (Figures 1A, B). At $18 \mathrm{mo}$ of age, there was significant splenomegaly (spleen weight $611 \mathrm{mg}$ vs $89 \mathrm{mg}, P<0.001$ ), increased total spleen cell counts (267 vs $153 \times 10^{6}$ cells per spleen, $P=0.031$ ), and decreased total marrow cell counts ( 23 vs $58 \times 10^{6}$ cells per femur, $\left.P<0.001\right)$ in the Tie2FF1 mice compared to age-matched Tie2-cre control mice (Figures 1C-E). Histology examination revealed extensive marrow osteopetrosis and destroyed splenic architecture, as well as increased fibrosis in both the marrow and spleen of the old Tie2FF1 mice compared to age matched control mice (Figures $\mathbf{1 F}-\mathbf{I}$ ). No evidence of leukemia transformation was observed in the Tie2FF1 mice. These findings indicate that the Tie2FF1 mice developed ET to PMF disease transformation with extramedullary splenic hematopoiesis during aging.

\section{Decreased Marrow Hematopoiesis During Aging in the Tie2FF1 Mice}

Previously, we and others reported that marrow HSCs were significantly expanded in young Tie2FF1 mice compared to agematched Tie2-cre control mice $(28,31,38)$. To examine how aging affects the neoplastic hematopoiesis in MPN, we first measured the numbers of marrow hematopoietic progenitor cells using colony formation assays. We found that the total hematopoietic progenitor cells were significantly increased in young (4-5mo) Tie2FF1 mice compared to age-matched control

FIGURE 1 | The Tie2FF1 mice develop ET to PMF transformation during aging. (A) Peripheral blood cell counts of Tie2FF1 (black line) and Tie2-cre control mice (grey line). ( $n=5-10$ mice in each group at $2 \mathrm{mo}$ and $4 \mathrm{mo} ; n=6-7$ mice in each group at $6 \mathrm{mo}, 12 \mathrm{mo}$, and $15 \mathrm{mo} ; n=10$ mice in each group at $18 \mathrm{mo}$ ). (B) Representative peripheral blood smear of 18mo old Tie2-cre and Tie2FF1 mice (40X magnification). (C-E) Spleen weight (C), total spleen cell counts (D), and total femur cell counts (E) in 18mo old Tie2-cre and Tie2FF1 mice ( $\mathrm{n=10}$ mice in each group). (F, G) Representative hematoxylin and eosin sections of marrow (F) and spleen (G) from 18mo old Tie2-cre and Tie2FF1 mice [F: 10X magnification, scale bar: 100uM; (G) 4X magnification, scale bar: 500uM]. (H, I) Representative reticulin stain of marrow (H) and spleen (I) from 18mo old Tie2-cre and Tie2FF1 mice (40X magnification). ${ }^{*} P<0.05$. 
mice (2.6-fold, $P=0.005)$; in contrast, there was no significant difference in progenitor cell numbers between old (18mo) Tie2FF1 mice and age-matched control mice (Figure 2A). Consistent with these findings, flow cytometry analysis revealed that marrow $\mathrm{Lin}^{-} \mathrm{CKit}^{+} \mathrm{Sca}{ }^{+} \mathrm{CD} 150^{+} \mathrm{CD} 48^{-}$HSCs (39) were significantly expanded in young Tie2FF1 mice compared to age-matched control mice $(9.7$-fold, $P<0.001)$, while there was no significant difference in HSC frequency between old Tie2FF1 and control mice $(0.125 \%$ vs. $0.184 \%, P=0.087)$ (Figure 2B). Considering that the total marrow cells were decreased 2.5 -fold in the old Tie2FF1 mice (Figure 1E), there was a 3.7-fold decrease in the absolute marrow HSC cell numbers in old Tie2FF1 mice compared to age-matched control mice.

To test whether the decreased phenotypic HSC number was associated with altered HSC function, we isolated marrow Lin HSPCs from Tie2-cre control and Tie2FF1 mice and measured their cell proliferation in vitro in serum-free liquid medium. At the end of a ten-day culture, while JAK2V617F mutant HSPCs from young Tie2FF1 mice displayed a higher proliferation rate than wild-type HSPCs from young control mice (1.6-fold, $P=0.012$ ), mutant HSPCs from old Tie2FF1 mice proliferated less than old control HSPCs (2.0-fold, $P=0.043$ ) (Figures 2C, D). Taken together, these data indicated that there was a loss of both HSPC number and HSPC function in the marrow of old Tie2FF1 mice during aging, mimicking the advanced phases of myelofibrosis (3).

\section{Expanded Splenic Extramedullary Hematopoiesis in the Tie2FF1 Mice}

Spleen is the most frequent organ involved in extramedullary hematopoiesis in patients with MPNs (3). To examine how the splenic hematopoiesis changes during aging and MPN disease progression in the old Tie2FF1 mice, we first measured the numbers of splenic hematopoietic progenitor cells using colony formation assays. We found that splenic hematopoietic progenitor cells were markedly increased in both young (19fold, $P<0.001)$ and old ( 19 -fold, $P<0.001)$ Tie2FF1 mice compared to age-matched control mice (Figure 3A). In line with this finding, flow cytometry analysis revealed that spleen HSCs were expanded in both young (53-fold $P=0.046$ ) and old (6.2-fold, $P=0.004)$ Tie2FF1 mice compared to age-matched control mice (Figure 3B). Considering that the total spleen cells were increased 1.7-fold in old Tie2FF1 mice (Figure 1D), there was a 10.5 -fold increase in the absolute spleen HSC numbers in old Tie2FF1 mice compared to age-matched control mice. When we isolated spleen Lin $^{-}$HSPCs from young and old Tie2-cre control and Tie2FF1 mice and cultured them in vitro, we found that both young (12.9-fold, $P=0.005)$ and old (4.3-fold, $P=0.022$ ) JAK2V617F mutant spleen HSPCs displayed a higher proliferation rate than age-matched wild-type control spleen HSPCs (Figures 3C, D). These results suggest that, in contrast to the decreased HSPC number and HSPC function we have observed in the marrow (Figure 2), the spleen of old Tie2FF1 mice was able to maintain the expansion of JAK2V617F mutant hematopoiesis during aging and MPN disease progression.

\section{Different HSC Functions in the Marrow and Spleen of Old Tie2FF1 Mice}

The differences between marrow (Figure 2) and spleen (Figure 3) hematopoiesis in the old Tie2FF1 mice prompted us to further investigate how aging and MPN disease progression
A

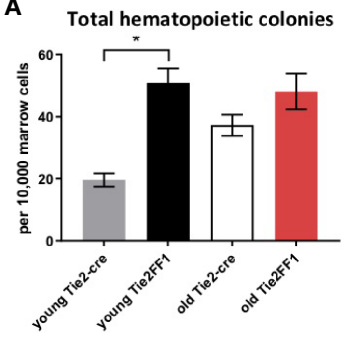

C

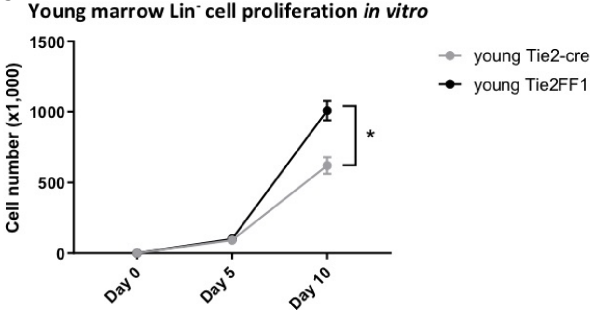

B
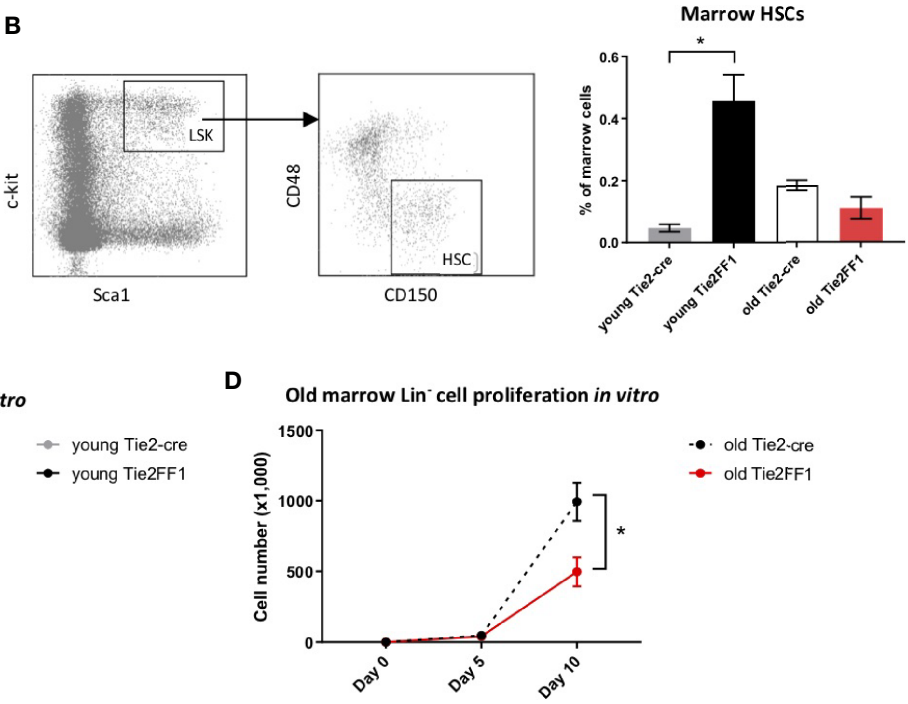

FIGURE 2 | Decreased marrow hematopoiesis in the Tie2FF1 mice during aging. (A) Colony formation assays in marrow cells isolated from young ( $\mathrm{n}=4$ mice in each group) and old ( $n=6-7$ mice in each group) Tie2-cre control and Tie2FF1 mice. (B) Representative flow cytometry plots showing gating strategy (left) of marrow Lin cKit ${ }^{+} \mathrm{Sca} 1^{+} \mathrm{CD} 150^{+} \mathrm{CD} 48^{-} \mathrm{HSCs}$ frequency (right) in young ( $\mathrm{n}=7$ mice in each group) and old ( $\mathrm{n}=5-6$ mice in each group) Tie2-cre control and Tie2FF1 mice. (C, D) Cell proliferation of marrow Lin- HSPCs isolated from young (C) and old (D) Tie2-cre control and Tie2FF1 mice. Cells were cultured on SFEM medium containing recombinant mouse SCF (10Ong/ $\mathrm{mL})$, recombinant mouse IL3 (6ng/ $\mathrm{mL})$, and recombinant human IL6 (1Ong/ $/ \mathrm{mL})$. Data are from one of two independent experiments (with triplicates in each experiment) that gave similar results. ${ }^{*} P<0.05$. 
A
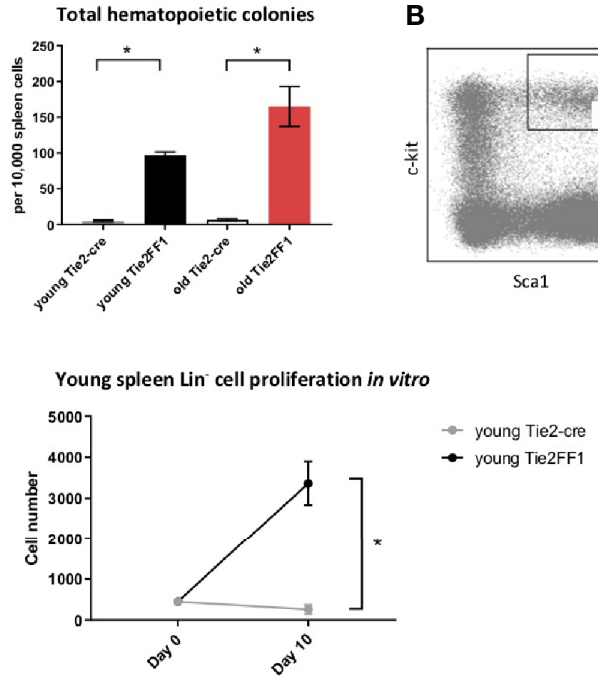

B

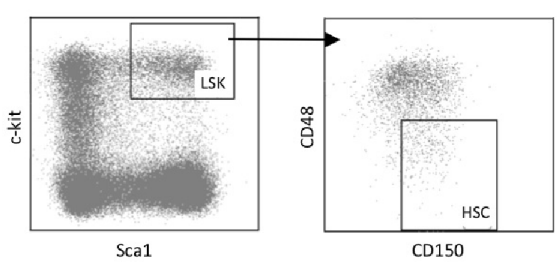

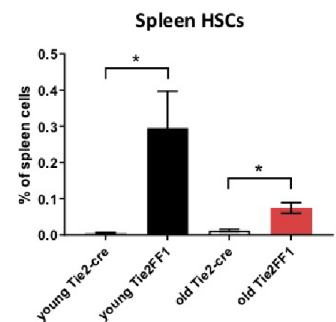

D

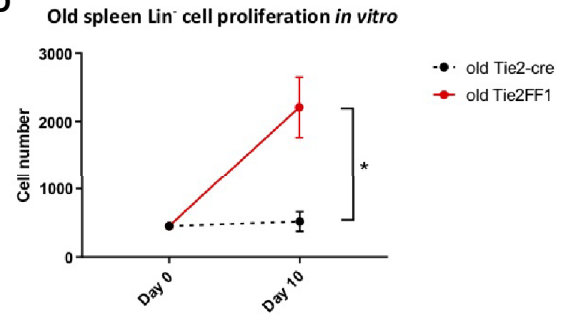

FIGURE 3 | Expanded splenic extramedullary hematopoiesis in the Tie2FF1 mice. (A) Colony formation assays in spleen cells isolated from young ( $\mathrm{n}=3$ mice in each group) and old ( $n=5-6$ mice in each group) Tie2-cre control and Tie2FF1 mice. (B) Spleen Lin-cKit ${ }^{+}$Sca $1^{+}$CD $150^{+} \mathrm{CD} 48^{-}$HSCs frequency in young ( $n=3$ mice in each group) and old ( $\mathrm{n}=5$ mice in each group) Tie2-cre control and Tie2FF1 mice. (C, D) Cell proliferation of spleen Lin HSPCs isolated from young (C) and old (D) Tie2cre control and Tie2FF1 mice. Cells were cultured in SFEM medium containing recombinant mouse SCF (10Ong/mL), recombinant mouse IL3 (6ng/mL), and recombinant human IL6 (1Ong/mL). Data are from one of two independent experiments (with triplicates in each experiment) that gave similar results. ${ }^{\star} \mathrm{P}<0.05$.

affect the JAK2V617F mutant HSC function differently in the marrow and spleen. First, we measured HSC proliferation in vivo by BrdU labeling (31). We found that JAK2V617F mutant HSCs from old Tie2FF1 mice proliferated more rapidly than wild-type HSCs from age-matched control mice in both the marrow $(58 \%$ vs $21 \%, P=0.001)$ and the spleen $(41 \%$ vs $22 \%, P=0.042)$ (Figures 4A, B). Next, we measured HSC cell apoptosis by assessing their activated caspase- 3 levels using flow cytometry analysis (31). We found that JAK2V617F mutant marrow HSCs from old Tie2FF1 mice displayed higher level of apoptosis compared to wild-type marrow HSCs from age-matched control mice $(3.0 \%$ vs $0.8 \%, P=0.006)$; in contrast, mutant spleen HSCs from old Tie2FF1 mice displayed significantly less apoptosis compared to wild-type spleen HSCs from control mice (1.8\% vs $11.6 \%, P=0.002)$ (Figures 4C, D). Since oncogenic mutation is a major stress to induce cellular senescence (40) and the JAK-STAT signaling has been reported to induce cellular senescence (41-44), we assessed HSC senescence by measuring their senescence associated $\beta$-galactosidase (SA- $\beta$-Gal) activity, which is a hallmark of cellular senescence (40). JAK2V617F mutant marrow HSCs from old Tie2FF1 mice demonstrated significantly higher senescence rates compared to wild-type marrow HSCs from age-matched control mice (18\% vs $9 \%$, $P=0.011$ ); in contrast, there was no difference in the cellular senescence rate between the mutant spleen HSCs from old Tie2FF1 mice and wild-type spleen HSCs from control mice (Figures 4E, F). Taken together, although the JAK2V617F mutant HSCs from old Tie2FF1 mice were more proliferative than wild-type HSCs in both the marrow and spleen, mutant HSCs were more apoptotic and senescent than wild-type HSCs in the marrow while the mutant cells were relatively protected in the spleen.

Most HSCs reside close to a perivascular niche in the marrow and spleen $(19,45)$. To understand how different vascular niches contribute to different HSC functions in the old Tie2FF1 mice, we measured marrow and spleen ECs (CD45-CD31+) by flow cytometry analysis. We found that marrow ECs were significantly decreased in old Tie2FF1 mice compared to agematched control mice; in contrast, spleen ECs were significantly expanded in old Tie2FF1 mice. These results were also confirmed by in vivo VE-cadherin labeling and immunofluorescence imaging of the marrow and spleen tissue samples (Figures 4G, H). In addition, while there was no difference in marrow EC senescence rate between old Tie2FF1 mice and old control mice, the JAK2V617F mutant splenic ECs from old Tie2FF1 mice were much less senescent compared to wild-type splenic ECs from age-matched control mice (Figures 4I, J). Therefore, the different vascular niche of the marrow and spleen could contribute to the decreased marrow hematopoiesis and expanded splenic hematopoiesis we have observed in the Tie2FF1 mice during aging.

\section{Persistent But Compensated Cardiomyopathy in the Old Tie2FF1 Mice}

Cardiovascular complications are the leading cause of morbidity and mortality in patients with MPNs. Previously, we reported that the Tie2FF1 mice developed spontaneous heart failure with thrombosis, vasculopathy, and cardiomyopathy at 20wk of age (32). Here, we followed the cardiovascular function of Tie2FF1 mice during aging. At $18 \mathrm{mo}$ of age, the Tie2FF1 mice continued to demonstrate a phenotype of dilated cardiomyopathy with a 
A
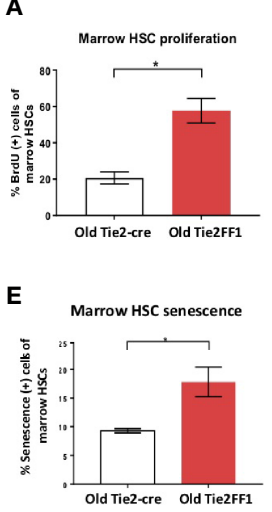

B

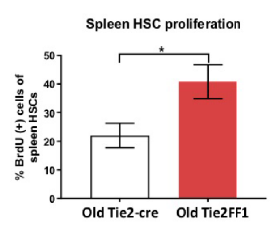

$\mathbf{F}$

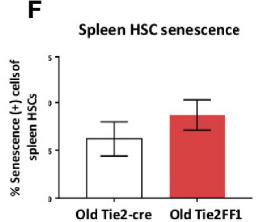

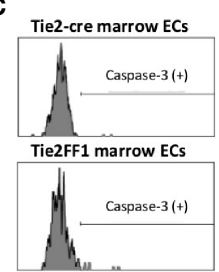

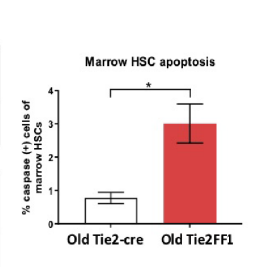

G

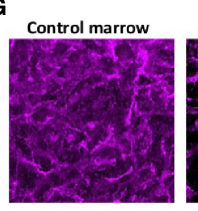

I
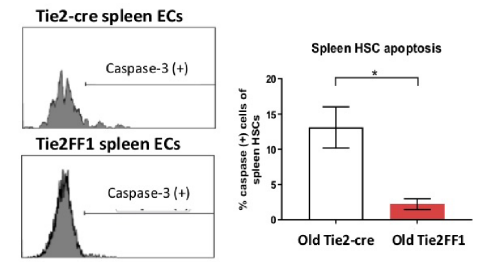

H
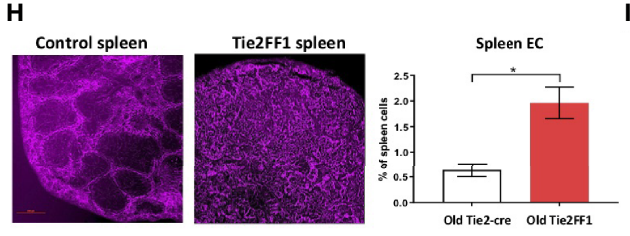
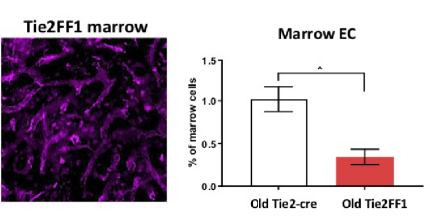

J
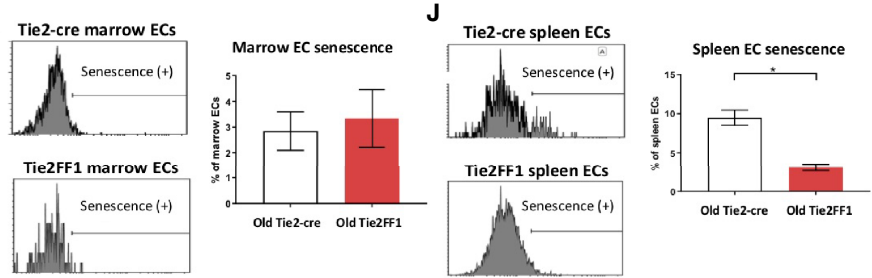

FIGURE 4 | Different HSC functions in the marrow and spleen of old Tie2FF1 mice (A, B) Cell proliferation rate of HSCs in the marrow (A) and spleen (B) measured by in vivo BrdU labeling (A: $n=4-5$ mice in each group; $B: n=4$ mice in each group). (C, D) Representative flow cytometry histograms (left) and quantitative analysis of cellular apoptosis rate of HSCs in the marrow (C) and spleen (D) measured by activated caspase-3 staining using flow cytometry analysis (C: $n=5$ mice in each group; $\mathbf{D}: n=5-6$ mice in each group). (E, F) Cellular senescence rate of HSCs in the marrow (E) and spleen (F) measured by SA- $\beta$-Gal activity using flow cytometry analysis $(\mathbf{E})$ : $n=5$ mice in each group; $(\mathbf{F}) n=5-6$ mice in each group). $(\mathbf{G}, \mathbf{H})$ Representative immunofluorescent images of VE cadherin $(+)$ vasculatures (magnification 10x for femur marrow and $4 \mathrm{x}$ for spleen) (left) and flow cytometry quantitative analysis of CD45 CD31 ${ }^{+} \mathrm{ECs}$ (right) of the marrow (G) and spleen (H) from $18 \mathrm{mo}$ Tie2cre control and Tie2FF1 mice (G): $n=8$ mice in each group; (H) $n=5$ mice in each group). (I) Representative flow cytometry histograms (left) and quantitative analysis of cellular senescence of marrow ECs ( $\mathrm{n}=4$ mice in each group). ( $\mathbf{J}$ ) Representative flow cytometry histograms (left) and quantitative analysis of cellular senescence of spleen ECs ( $n=3$ mice in each group). ${ }^{*} P<0.05$.

moderate but significant decrease in LV EF (56\% versus $66 \%$, $P=0.043)$, an increase in $\mathrm{LV}$ end-diastolic volume $(84 \mu \mathrm{L}$ vs $71 \mu \mathrm{L}$, $P=0.086$ ) and end-systolic volume ( $38 \mu \mathrm{L}$ vs $25 \mu \mathrm{L}, P=0.041$ ), and an increase in $\mathrm{LV}$ mass $(156 \mathrm{mg}$ vs $113 \mathrm{mg}, P=0.021$ ) compared to age-matched control mice (Figure 5A). Pathological evaluation confirmed the diagnosis of dilated cardiomyopathy in old Tie2FF1 mice with significantly increased heart weight-to-tibia length ratio compared to age-matched control mice (0.014 vs $0.011 \mathrm{gram} / \mathrm{mm}, P=0.004$ ) (Figure 5B). Increased lung weight in old Tie2FF1 mice compared to control mice ( 0.287 vs 0.226 gram) further indicated the presence of pulmonary edema commonly associated with heart failure (Figure 5C). Similar to what we previously reported in the young Tie2FF1 mice (32), there was spontaneous thrombosis in the right ventricle and pulmonary arteries in the old Tie2FF1 mice, while age-matched Tie2-cre control mice had no evidence of spontaneous thrombosis in their heart or lungs (Figures 5D, E). Despite these cardiovascular dysfunctions, there was no difference in body weight between old Tie2FF1 mice and control mice (Figure 5F), nor was there any significantly increased incidence of sudden death in the old Tie2FF1 mice compared to age-matched control mice. These findings suggested that there was a persistent but compensated cardiomyopathy and heart failure in the Tie2FF1 mice during aging.

\section{DISCUSSIONS}

With heterozygous human JAK2V617F transgene expression in both the hematopoietic cells and vascular ECs, the Tie2FF1 mice developed an ET-like phenotype at young age (2mo old) which transformed to PMF during aging. The mice also demonstrated features of extramedullary splenic hematopoiesis, spontaneous vascular thrombosis, cardiovascular dysfunction, which persisted during the aging process. Compared to other MPN murine models reported so far (7-18), the Tie2FF1 mice is the first MPN murine model that faithfully recapitulated almost all the key features of the human MPN diseases. Considering the presence of the JAK2V617F mutation in microvascular ECs isolated from patients with MPNs $(21,22,26)$ and the recapitulation of all the key features of human MPN diseases by the Tie2FF1 mice, the roles of endothelial dysfunction in the hematologic and cardiovascular pathogenesis of MPN and whether the MPN vascular niche can be targeted to provide more effective therapeutic strategies for patients with these diseases shall be further investigated.

Extramedullary splenic hematopoiesis often compensates for normal hematopoietic suppression in neoplastic conditions (46, 47). Splenomegaly is a common feature in patients with MPNs as a result of extramedullary hematopoiesis, in which HSCs 
A

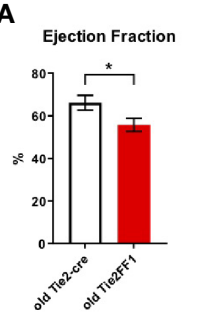

D

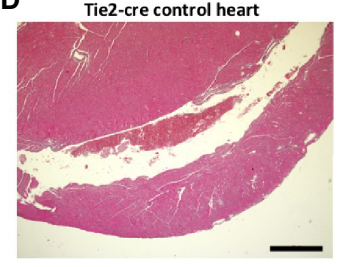

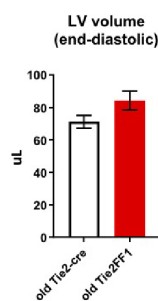

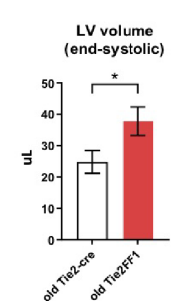

Tie2FF1 heart

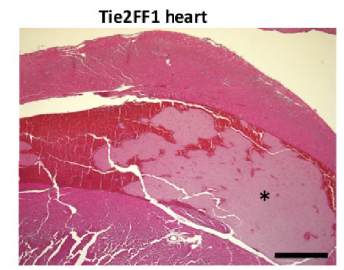

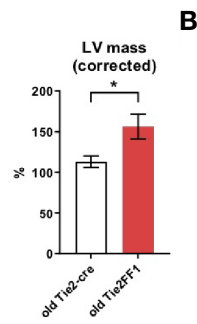

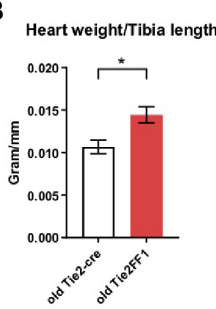

E

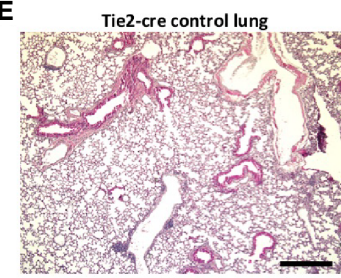

C

F Body weight
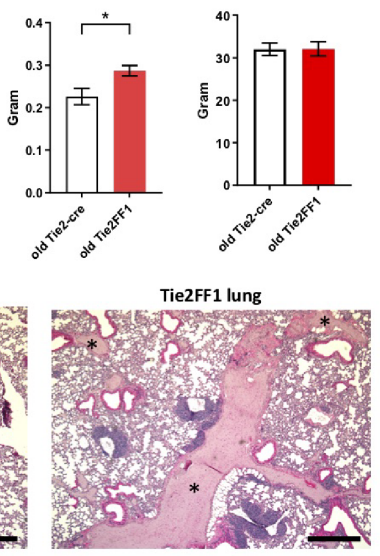

FIGURE 5 | Persistent cardiomyopathy and congestive heart failure in the old Tie2FF1 mice. (A) Measurements of left ventricular (LV) ejection fraction, end-diastolic and end-systolic volume, and mass by transthoracic echocardiography in 18mo old Tie2-cre control and Tie2FF1 mice. (B) Heart weight adjusted by tibia length of 18mo old Tie2-cre control and Tie2FF1 mice. (C) Lung weight of 18mo old Tie2-cre control and Tie2FF1 mice. (D) Representative H\&E staining of transverse sections of heart from Tie2-cre control and Tie2FF1 mice. Note the presence of thrombus (*) in right ventricle (magnification 4x, scale bar: 500uM). (E) Representative H\&E staining of coronal sections of lung from Tie2-cre control and Tie2FF1 mice. Note the presence of thrombus (star*) in segment pulmonary arteries of the Tie2FF1 mice (magnification 4x, scale bar: 500uM). (F) Body weight of 18mo old Tie2-cre control and Tie2FF1 mice. $n=8-11$ mice in each group. ${ }^{\star} P<0.05$.

are mobilized to sites outside the marrow to expand hematopoiesis. Our study showed that the extramedullary splenic hematopoiesis could also compensate/maintain MPN neoplastic hematopoiesis during disease transformation/ progression. Together with a previous report that the spleens of PMF patients contain the neoplastic stem cells for MPN development (48), these findings indicate that effective targeting of the splenic neoplastic hematopoiesis might be necessary for successful MPN therapies.

ECs are an essential component of the perivascular niche in the marrow and spleen $(19,45)$. It is known that vascular ECs within different tissues have unique gene expression profile and cellular function (49). Results from our previous studies and current work demonstrated that there was a significant heterogeneity of the JAK2V617F mutant ECs in different parts of the circulation (e.g., marrow, spleen, heart32). How the same JAK2V617F mutation results in different EC functions in different tissues is not fully understood. Since flow shear stress has key roles in endothelial function (50) and biomechanical forces can regulate HSC function (51), it is possible that different e.g. flow rate, shear stress, or hydrostatic pressure in different tissues can contribute to different JAK2V617F -bearing EC functions.

In summary, our previous (27-32) and current work have demonstrated that JAK2V617F-bearing vascular ECs play an important role in both the hematologic and cardiovascular disease processes of MPNs. Results from our studies also revealed a significant heterogeneity of the JAK2V617F mutant ECs in different parts of the circulation and the spleen is functionally important for MPN neoplastic hematopoiesis during aging and disease progression. Therefore, the Tie2FF1 mice provide a unique in vivo model to screen or test potential preventive and therapeutic interventions for patients with MPNs.

\section{DATA AVAILABILITY STATEMENT}

The datasets presented in this article are not readily available because none. Requests to access the datasets should be directed to Huichun.zhan@stonybrookmedicine.edu.

\section{ETHICS STATEMENT}

The animal study was reviewed and approved by Stony Brook Institutional Animal Care and Use Committee

\section{AUTHOR CONTRIBUTIONS}

HZhang performed various in vitro and in vivo experiments of the project, and analyzed the data. AY assisted various in vitro culture experiments. SL provided technical assistance in various parts of the project. HZhan conceived the projects, analyzed the data, interpreted the results, and wrote the manuscript. All authors contributed to the article and approved the submitted version.

\section{FUNDING}

This research was supported by the National Heart, Lung, and Blood Institute grant NIH R01 HL134970 (HZhan) and VA Merit Award BX003947 (HZhang). 


\section{REFERENCES}

1. Landolfi R, Di Gennaro L, Falanga A. Thrombosis in Myeloproliferative Disorders: Pathogenetic Facts and Speculation. Leukemia (2008) 22:2020-8. doi: 10.1038/leu.2008.253

2. Zhan H, Spivak JL. The Diagnosis and Management of Polycythemia Vera, Essential Thrombocythemia, and Primary Myelofibrosis in the JAK2 V617F Era. Clin Adv Hematol Oncol (2009) 7:334-42.

3. Spivak JL. Myeloproliferative Neoplasms. N Engl J Med (2017) 376:2168-81. doi: 10.1056/NEJMra1406186

4. Stein BL, Oh ST, Berenzon D, Hobbs GS, Kremyanskaya M, Rampal RK, et al. Polycythemia Vera: An Appraisal of the Biology and Management 10 Years After the Discovery of JAK2 V617F. J Clin Oncol (2015) 33:3953-60. doi: 10.1200/JCO.2015.61.6474

5. Nangalia J, Green AR. Myeloproliferative Neoplasms: From Origins to Outcomes. Hematol Am Soc Hematol Educ Program (2017) 2017:470-9. doi: 10.1182/asheducation-2017.1.470

6. Dunbar A, Nazir A, Levine R. Overview of Transgenic Mouse Models of Myeloproliferative Neoplasms (MPNs). Curr Protoc Pharmacol (2017) 77:14 40 1-14 40 19. doi: 10.1002/cpph.23

7. Bumm TG, Elsea C, Corbin AS, d M, Sherbenou D, Wood L, et al. Characterization of Murine JAK2V617F-Positive Myeloproliferative Disease. Cancer Res (2006) 66:11156-65. doi: 10.1158/0008-5472.CAN-06-2210

8. Lacout C, Pisani DF, Tulliez M, Gachelin FM, Vainchenker W, Villeval JL. JAK2V617F Expression in Murine Hematopoietic Cells Leads to MPD Mimicking Human PV With Secondary Myelofibrosis. Blood (2006) 108:1652-60. doi: 10.1182/blood-2006-02-002030

9. Wernig G, Mercher T, Okabe R, Levine RL, Lee BH, Gilliland DG. Expression of Jak2V617F Causes a Polycythemia Vera-Like Disease With Associated Myelofibrosis in a Murine Bone Marrow Transplant Model. Blood (2006) 107:4274-81. doi: 10.1182/blood-2005-12-4824

10. Zaleskas VM, Krause DS, Lazarides K, Patel N, Hu Y, Li S, et al. Molecular Pathogenesis and Therapy of Polycythemia Induced in Mice by JAK2 V617F. PloS One (2006) 1:e18. doi: 10.1371/journal.pone.0000018

11. Shide K, Shimoda HK, Kumano T, Karube K, Kameda T, Takenaka K, et al. Development of ET, Primary Myelofibrosis and PV in Mice Expressing JAK2 V617F. Leukemia (2008) 22:87-95. doi: 10.1038/sj.leu.2405043

12. Tiedt R, Hao-Shen H, Sobas MA, Looser R, Dirnhofer S, Schwaller J, et al. Ratio of Mutant JAK2-V617F to Wild-Type Jak2 Determines the MPD Phenotypes in Transgenic Mice. Blood (2008) 111:3931-40. doi: 10.1182/ blood-2007-08-107748

13. Xing S, Wanting TH, Zhao W, Ma J, Wang S, Xu X, et al. Transgenic Expression of JAK2V617F Causes Myeloproliferative Disorders in Mice. Blood (2008) 111:5109-17. doi: 10.1182/blood-2007-05-091579

14. Akada H, Yan D, Zou H, Fiering S, Hutchison RE, Mohi MG. Conditional Expression of Heterozygous or Homozygous Jak2V617F From Its Endogenous Promoter Induces a Polycythemia Vera-Like Disease. Blood (2010) 115:3589-97. doi: 10.1182/blood-2009-04-215848

15. Mullally A, Lane SW, Ball B, Megerdichian C, Okabe R, Al-Shahrour F, et al. Physiological Jak2V617F Expression Causes a Lethal Myeloproliferative Neoplasm With Differential Effects on Hematopoietic Stem and Progenitor Cells. Cancer Cell (2010) 17:584-96. doi: 10.1016/j.ccr.2010.05.015

16. Marty C, Lacout C, Martin A, Hasan S, Jacquot S, Birling MC, et al. Myeloproliferative Neoplasm Induced by Constitutive Expression of JAK2V617F in Knock-in Mice. Blood (2010) 116:783-7. doi: 10.1182/blood2009-12-257063

17. Li J, Spensberger D, Ahn JS, Anand S, Beer PA, Ghevaert C, et al. JAK2 V617F Impairs Hematopoietic Stem Cell Function in a Conditional Knock-in Mouse Model of JAK2 V617F-Positive Essential Thrombocythemia. Blood (2010) 116:1528-38. doi: 10.1182/blood-2009-12-259747

18. Li J, Kent DG, Godfrey AL, Manning H, Nangalia J, Aziz A, et al. JAK2V617F Homozygosity Drives a Phenotypic Switch in Myeloproliferative Neoplasms, But Is Insufficient to Sustain Disease. Blood (2014) 123:3139-51. doi: 10.1182/ blood-2013-06-510222

19. Crane GM, Jeffery E, Morrison SJ. Adult Haematopoietic Stem Cell Niches. Nat Rev Immunol (2017) 17:573-90. doi: 10.1038/nri.2017.53

20. Wu KK, Thiagarajan P. Role of Endothelium in Thrombosis and Hemostasis. Annu Rev Med (1996) 47:315-31. doi: 10.1146/annurev.med.47.1.315
21. Sozer S, Fiel MI, Schiano T, Xu M, Mascarenhas J, Hoffman R. The Presence of JAK2V617F Mutation in the Liver Endothelial Cells of Patients With BuddChiari Syndrome. Blood (2009) 113:5246-9. doi: 10.1182/blood-2008-11-191544

22. Rosti V, Villani L, Riboni R, Riboni R, Poletto V, Bonetti E, Tozzi, et al. Spleen Endothelial Cells From Patients With Myelofibrosis Harbor the JAK2V617F Mutation. Blood (2013) 121:360-8. doi: 10.1182/blood-2012-01-404889

23. Yoder MC, Mead LE, Prater D, Krier TR, Mroueh KN, Li F, et al. Redefining Endothelial Progenitor Cells via Clonal Analysis and Hematopoietic Stem/ Progenitor Cell Principals. Blood (2007) 109:1801-9. doi: 10.1182/blood2006-08-043471

24. Teofili L, Martini M, Iachininoto MG, Capodimonti S, Nuzzolo ER, Torti L, et al. Endothelial Progenitor Cells Are Clonal and Exhibit the JAK2(V617F) Mutation in a Subset of Thrombotic Patients With $\mathrm{Ph}$-Negative Myeloproliferative Neoplasms. Blood (2011) 117:2700-7. doi: 10.1182/ blood-2010-07-297598

25. Piaggio G, Rosti V, Corselli M, Bertolotti F, Bergamaschi G, Pozzi S, et al. Endothelial Colony-Forming Cells From Patients With Chronic Myeloproliferative Disorders Lack the Disease-Specific Molecular Clonality Marker. Blood (2009) 114:3127-30. doi: 10.1182/blood-2008-12-190991

26. Helman R, Pereira WO, Marti LC, Campregher PV, Puga RD, Hamerschlak N, et al. Granulocyte Whole Exome Sequencing and Endothelial JAK2V617F in Patients With JAK2V617F Positive Budd-Chiari Syndrome Without Myeloproliferative Neoplasm. Br J Haematol (2018) 180:443-5. doi: 10.1111/bjh.14327

27. Lin CH, Kaushansky K, Zhan H. JAK2V617F-Mutant Vascular Niche Contributes to JAK2V617F Clonal Expansion in Myeloproliferative Neoplasms. Blood Cells Mol Dis (2016) 62:42-8. doi: 10.1016/j.bcmd. 2016.09.004

28. Zhan H, Lin CHS, Segal Y, Kaushansky K. The JAK2V617F-Bearing Vascular Niche Promotes Clonal Expansion in Myeloproliferative Neoplasms. Leukemia (2018) 32:462-9. doi: 10.1038/leu.2017.233

29. Lin CHS, Zhang Y, Kaushansky K, Zhan H. JAK2V617F-Bearing Vascular Niche Enhances Malignant Hematopoietic Regeneration Following Radiation Injury. Haematologica (2018) 103:1160-8. doi: 10.3324/haematol.2017.185736

30. Mazzeo C, Quan M, Wong H, Castiglione M, Kaushansky K, Zhan H. JAK2V617F Mutant Endothelial Cells Promote Neoplastic Hematopoiesis in a Mixed Vascular Microenvironment. Blood Cells Mol Dis (2021) 90:102585. doi: 10.1016/j.bcmd.2021.102585

31. Castiglione M, Zhang H, Kaushansky K, Zhan H. Cell Competition Between Wild-Type and JAK2V617F Mutant Cells in a Murine Model of a Myeloproliferative Neoplasm. Exp Hematol (2021). doi: 10.1101/ 2020.08.26.267070

32. Castiglione M, Jiang YP, Mazzeo C, Lee S, Chen JS, Kaushansky K, et al. Endothelial JAK2V617F Mutation Leads to Thrombosis, Vasculopathy, and Cardiomyopathy in a Murine Model of Myeloproliferative Neoplasm. J Thromb Haemost (2020). doi: 10.1101/2019.12.31.891721

33. Constien R, Forde A, Liliensiek B, Grone HJ, Nawroth P, Hammerling G, et al. Characterization of a Novel EGFP Reporter Mouse to Monitor Cre Recombination as Demonstrated by a Tie2 Cre Mouse Line. Genesis (2001) 30:36-44. doi: 10.1002/gene.1030

34. Zhan H, Ma Y, Lin CH, Kaushansky K. JAK2V617F-Mutant Megakaryocytes Contribute to Hematopoietic Stem/Progenitor Cell Expansion in a Model of Murine Myeloproliferation. Leukemia (2016) 30:2332-41. doi: 10.1038/ leu.2016.114

35. Guo P, Poulos MG, Palikuqi B, Badwe CR, Lis R, Kunar B, et al. Endothelial Jagged-2 Sustains Hematopoietic Stem and Progenitor Reconstitution After Myelosuppression. J Clin Invest (2017) 127:4242-56. doi: 10.1172/JCI92309

36. Gao S, Ho D, Vatner DE, Vatner SF. Echocardiography in Mice. Curr Protoc Mouse Biol (2011) 1:71-83. doi: 10.1002/9780470942390.mo100130

37. Etheridge SL, Roh ME, Cosgrove ME, Sangkhae V, Fox NE, Chen J, et al. JAK2V617F-Positive Endothelial Cells Contribute to Clotting Abnormalities in Myeloproliferative Neoplasms. Proc Natl Acad Sci USA (2014) 111:2295300. doi: 0.1073/pnas.1312148111

38. Sangkhae V, Etheridge SL, Kaushansky K, Hitchcock IS. The Thrombopoietin Receptor, MPL, Is Critical for Development of a JAK2V617F-Induced Myeloproliferative Neoplasm. Blood (2014) 124:3956-63. doi: 10.1182/ blood-2014-07-587238 
39. Kiel MJ, Yilmaz OH, Iwashita T, Yilmaz OH, Terhorst C, Morrison SJ. SLAM Family Receptors Distinguish Hematopoietic Stem and Progenitor Cells and Reveal Endothelial Niches for Stem Cells. Cell (2005) 121:1109-21. doi: 10.1016/j.cell.2005.05.026

40. Sharpless NE, Sherr CJ. Forging a Signature of In Vivo Senescence. Nat Rev Cancer (2015) 15:397-408. doi: 10.1038/nrc3960

41. Xu M, Tchkonia T, Ding H, Ogrodnik M, Lubbers ER, Pirtskhalava T, et al. JAK Inhibition Alleviates the Cellular Senescence-Associated Secretory Phenotype and Frailty in Old Age. Proc Natl Acad Sci USA (2015) 112: E6301-10. doi: 10.1073/pnas.1515386112

42. Xu M, Palmer AK, Ding H, Weivoda MM, Pirtskhalava T, White TA, et al. Targeting Senescent Cells Enhances Adipogenesis and Metabolic Function in Old Age. Elife (2015) 4:e12997. doi: 10.7554/eLife.12997

43. Novakova Z, Hubackova S, Kosar M, Janderova-Rossmeislova L, Dobrovolna J, Vasicova P, et al. Cytokine Expression and Signaling in Drug-Induced Cellular Senescence. Oncogene (2010) 29:273-84. doi: 10.1038/onc.2009.318

44. Griveau A, Wiel C, Ziegler DV, Bergo MO, Bernard D. The JAK1/2 Inhibitor Ruxolitinib Delays Premature Aging Phenotypes. Aging Cell (2020) 19:e13122. doi: 10.1111 /acel.13122

45. Inra CN, Zhou BO, Acar M, Murphy MM, Richardson J, Zhao Z, et al. A Perisinusoidal Niche for Extramedullary Haematopoiesis in the Spleen. Nature (2015) 527:466-71. doi: 10.1038/nature15530

46. Cheng H, Hao S, Liu Y, Pang Y, Ma S, Dong F, et al. Leukemic Marrow Infiltration Reveals a Novel Role for Egr3 as a Potent Inhibitor of Normal Hematopoietic Stem Cell Proliferation. Blood (2015) 126:1302-13. doi: 10.1182/ blood-2015-01-623645

47. Zhang TY, Dutta R, Benard B, Zhao F, Yin R, Majeti R. IL-6 Blockade Reverses Bone Marrow Failure Induced by Human Acute Myeloid Leukemia. Sci Transl Med (2020) 12:538. doi: 10.1126/scitranslmed.aax5104
48. Wang X, Prakash S, Lu M, Tripodi J, Ye F, Najfeld V, et al. Spleens of Myelofibrosis Patients Contain Malignant Hematopoietic Stem Cells. J Clin Invest (2012) 122:3888-99. doi: 10.1172/JCI64397

49. Nolan DJ, Ginsberg M, Israely E, Palikuqi B, Poulos MG, James D, et al. Molecular Signatures of Tissue-Specific Microvascular Endothelial Cell Heterogeneity in Organ Maintenance and Regeneration. Dev Cell (2013) 26:204-19. doi: 10.1016/j.devcel.2013.06.017

50. Chiu JJ, Chien S. Effects of Disturbed Flow on Vascular Endothelium: Pathophysiological Basis and Clinical Perspectives. Physiol Rev (2011) 91:327-87. doi: 10.1152/physrev.00047.2009

51. Lee HJ, Li N, Evans SM, Diaz MF, Wenzel PL. Biomechanical Force in Blood Development: Extrinsic Physical Cues Drive Pro-Hematopoietic Signaling Differentiation (2013) 86:92-103. doi: 10.1016/j.diff.2013.06.004

Conflict of Interest: The authors declare that the research was conducted in the absence of any commercial or financial relationships that could be construed as a potential conflict of interest.

Publisher's Note: All claims expressed in this article are solely those of the authors and do not necessarily represent those of their affiliated organizations, or those of the publisher, the editors and the reviewers. Any product that may be evaluated in this article, or claim that may be made by its manufacturer, is not guaranteed or endorsed by the publisher.

Copyright (c) 2021 Zhang, Yeware, Lee and Zhan. This is an open-access article distributed under the terms of the Creative Commons Attribution License (CC BY). The use, distribution or reproduction in other forums is permitted, provided the original author(s) and the copyright owner(s) are credited and that the original publication in this journal is cited, in accordance with accepted academic practice. No use, distribution or reproduction is permitted which does not comply with these terms. 